\title{
Relative serum amyloid A (SAA) values: the influence of SAA1 genotypes and corticosteroid treatment in Japanese patients with rheumatoid arthritis
}

\author{
T Yamada, Y Okuda, K Takasugi, K Itoh, J Igari
}

\begin{abstract}
Objectives-(1) To determine whether serum concentration of serum amyloid $A$ (SAA) protein is influenced by the SAA1 allele in Japanese patients with rheumatoid arthritis (RA) as previously shown in a healthy control group; and (2) to analyse what factors, based on such an allelic bias, influence the relative SAA values of those patients.

Methods-SAA and $\mathrm{C}$ reactive protein (CRP) concentrations together with SAA1 genotypes were determined in 316 Japanese patients with RA. The relative SAA values were evaluated as an SAA/CRP ratio.

Results-Comparison of the three SAA1 homozygote groups showed that the SAA/ CRP ratio was highest in the $1.5 / 1.5$ group (mean 9.0, p $<0.01 v$ the other two homozygote groups) followed by the $1.3 / 1.3$ group (mean 7.2, NS $v$ the 1.1/1.1 group) and the 1.1/1.1 group (mean 4.0). The SAA/CRP ratio was significantly higher in patients receiving corticosteroids regardless of the presence of allele 1.5. No clear differences in the ratio between patients with or without amyloidosis were found.

Conclusion-The SAA1.5 allele and corticosteroid treatment had a positive influ-

ence on SAA concentrations in serum.
These findings are important when evaluating SAA concentration in inflammatory diseases and when considering the cause or treatment of amyloidosis. (Ann Rheum Dis 2001;60:124-127)
\end{abstract}

Department of Clinical Pathology, Juntendo University School of Medicine, Tokyo, Japan T Yamada

J Igari

Centre for Rheumatic Diseases, Dohgo Spa Hospital, Matsuyama, Japan

Y Okuda

K Takasugi

Department of Clinical Laboratory Medicine, Jichi Medical School, Tochigi, Japan

K Itoh

Correspondence to: Dr T Yamada, Department of Clinical Pathology, Juntendo University School of Medicine, 2-1-1 Hongo, Bunkyo-ku, Tokyo,

113-8421 Japan

toshiyam@med.juntendo.ac.jp

Accepted 7 June 2000
Serum amyloid A (SAA) and C reactive protein (CRP) are the most sensitive acute phase proteins in plasma. ${ }^{12}$ Plasma concentrations of these proteins increase up to 1000 -fold of their physiological levels in response to inflammation. In plasma both SAA and CRP are believed to be derived mostly from the liver. Hepatocytes secrete these proteins by the induction of inflammation related cytokines such as tumour necrosis factor $\alpha$, interleukin 1 , and interleukin $6 .{ }^{3}$ Although some differences in cytokine regulation by hepatocytes has been noted between SAA and CRP, ${ }^{4}$ clinical observations suggest that plasma concentrations of these two proteins are highly correlated with each other in most inflammatory diseases. ${ }^{5}{ }^{6} \mathrm{In}$ some conditions, such as viral infection, ${ }^{7}$ rejection of renal allograft, ${ }^{8}$ and therapeutic use of corticosteroids, ${ }^{9}$ SAA values seem to be relatively high, whereas those of CRP are relatively low, but the reason is unknown.

Polymorphism has not been noted in human CRP, whereas SAA proteins consist of several genetically determined isotypes. ${ }^{10-12}$ Acute phase isotypes of human SAA are encoded at three different loci, SAA1, SAA2, and SAA3; SAA3 is a pseudogene. The allelic variants are six in SAA1 and two in SAA2 loci, respectively. ${ }^{12}$ In a Japanese population the frequencies of alleles 1.1, 1.3, and 1.5 are equal, whereas 1.2 is rare $(1-2 \%) .^{13}$

SAA is a serum precursor of amyloid A (AA) protein, the fibrillar component in reactive amyloid deposits. ${ }^{14}$ Reactive amyloidosis is a potentially fatal complication in chronic inflammatory disorders. ${ }^{15}{ }^{16}$ Approximately 10\% of patients with rheumatoid arthritis (RA) are believed to have amyloid deposits, according to a study concerning gastroendoscopic biopsies. ${ }^{17}{ }^{18}$ A prolonged high plasma level of SAA in chronic inflammation may lead to the deposition of its degraded products, AA proteins, in tissues. However, a high concentration of SAA is not sufficient for the development of amyloidosis. Recent studies have focused on the polymorphism of SAA as a genetic background for amyloidogenesis. ${ }^{19-21}$ SAA1 allele frequency has been analysed because this isotype is predominant in plasma, and AA proteins are derived largely from it. ${ }^{22}$ The alleles 1.1 and 1.3 have been proposed as positive risk factors in white and Japanese patients, respectively. ${ }^{19-21}$ It is currently unknown how a specific SAA1 allele is linked to the development of amyloidosis.

Recently, our group has found that the SAA1 allele influences plasma concentration of SAA. In the Japanese population, subjects with the SAA1.5 allele have a higher plasma SAA concentration than those without it. ${ }^{23}$ This may be a significant finding for the amyloidogenicity of SAA and also for the biology of the protein. Because the previous study was conducted on an apparently healthy population, this study assessed whether such an influence was seen in patients with raised SAA. Patients with RA were selected for this purpose. SAA values were evaluated using the ratio SAA/ CRP. Taking such an allelic bias into consideration, we investigated what factors, including the use of corticosteroids, affect the ratio. Further, we examined the relation between allele regulated SAA concentration and the development of amyloidosis. 
Table 1 Clinical profiles of Fapanese patients classified by SAA1 genotype

\begin{tabular}{|c|c|c|c|c|c|c|c|c|c|c|c|}
\hline \multirow{2}{*}{$\begin{array}{l}\text { SAA1 } \\
\text { genotype }\end{array}$} & \multirow{2}{*}{$\begin{array}{l}\text { No } \\
\text { (male) }\end{array}$} & \multirow[b]{2}{*}{ Aget } & \multicolumn{4}{|c|}{ Class* } & \multirow{2}{*}{$\begin{array}{l}\text { Durationt } \\
\text { (years) }\end{array}$} & \multirow{2}{*}{$\begin{array}{l}\text { Amyloidosis } \\
(\text { No }(\%))\end{array}$} & \multicolumn{3}{|c|}{ Treatment (No (\%)) } \\
\hline & & & $I$ & $I I$ & $I I I$ & $I V$ & & & $C S \ddagger$ & $D M \ddagger$ & $I S \ddagger$ \\
\hline $1.1 / 1.1$ & 27 (3) & 63.4 & 2 & 12 & 10 & 3 & 17.6 & $1(4)$ & $19(70)$ & $23(85)$ & $5(19)$ \\
\hline $1.1 / 1.2$ & $3(1)$ & 53.0 & 0 & 2 & 1 & 0 & 11.0 & $0(0)$ & $2(67)$ & $3(100)$ & $2(67)$ \\
\hline $1.1 / 1.3$ & $73(11)$ & 65.5 & 4 & 33 & 33 & 3 & 17.6 & $8(11)$ & $55(75)$ & $59(81)$ & $41(56)$ \\
\hline $1.1 / 1.5$ & $55(5)$ & 63.2 & 6 & 18 & 30 & 1 & 19.2 & $13(24)$ & $43(78)$ & $44(80)$ & $22(40)$ \\
\hline $1.2 / 1.3$ & $4(0)$ & 57.3 & 0 & 3 & 1 & 0 & 9.8 & $0(0)$ & $3(75)$ & $4(100)$ & $0(0)$ \\
\hline $1.2 / 1.5$ & $3(0)$ & 66.3 & 0 & 2 & 1 & 0 & 17.3 & $0(0)$ & $2(67)$ & $3(100)$ & $3(100)$ \\
\hline $1.3 / 1.3$ & $44(4)$ & 64.6 & 0 & 14 & 28 & 2 & 17.8 & $13(30)$ & $37(84)$ & $33(75)$ & $13(30)$ \\
\hline $1.3 / 1.5$ & $78(11)$ & 67.6 & 5 & 32 & 39 & 2 & 17.4 & $25(32)$ & $65(83)$ & $58(74)$ & $20(26)$ \\
\hline $1.5 / 1.5$ & $29(3)$ & 66.7 & 2 & 10 & 17 & 0 & 17.7 & $6(21)$ & $23(79)$ & $25(86)$ & $15(52)$ \\
\hline Total & $316(38)$ & 65.2 & & & & & 17.7 & $66(21)$ & $249(79)$ & $252(80)$ & $121(38)$ \\
\hline
\end{tabular}

${ }^{\star}$ Class $=$ Steinbrocker functional class.

tAge and duration are mean values.

$\ddagger C S$ = corticosteroid; DM = DMARDs; IS = immunosuppressive agents.

\section{Methods}

PATIENTS

Three hundred and sixteen Japanese subjects (38 male, 278 female, aged 16-89), both inpatients and outpatients at the Centre for Rheumatism, Dohgo Spa Hospital, Matsuyama, Japan during the months of June and July 1999, took part in this study. Informed consent was obtained from all subjects. All patients met the 1987 revised RA criteria of the American Rheumatism Association. ${ }^{24}$ All patients had undergone gastroduodenal endoscopic biopsy within the past two years; amyloidosis was diagnosed histologically from the biopsy specimens. Therapeutic drugs were grouped into three categories: corticosteroids, disease modifying antirheumatic drugs (DMARDs), and immunosuppressive agents. Corticosteroid was given as oral prednisolone. DMARDs included intramuscular gold, D-penicillamine, auranofin, bucillamine, salazosulfapyridine, and actarit (4-acetylaminophenylacetic acid). Immunosuppressive agents included methotrexate, azathioprine, cyclophosphamide, and tacrolimus (FK-506). DMARDs or immunosuppressive agents were given according to the commonly recommended regimen, and doses of corticosteroids varied among subjects. Table 1 shows the frequency of use.

ANALYSIS

Blood was drawn from inpatients at the monthly examination and from outpatients at the regular visit. SAA1 genotypes were determined using the genomic DNA of the patients by a polymerase chain reaction-restriction fragment length polymorphism analysis as described elsewhere. ${ }^{23}$ SAA and CRP concentrations in serum were determined by a latex agglutination turbidimetric immunoassay, ${ }^{25}$ which is currently available from Eiken Chemicals Co Ltd, Tokyo, Japan. A statistical comparison of data between the groups was carried out with a $\chi^{2}$ test or Mann-Whitney U test.

Table 2 SAA1 allele frequency and amyloid involvement

\begin{tabular}{llll}
\hline \multirow{4}{*}{$\begin{array}{l}\text { SAA1 } \\
\text { allele }\end{array}$} & \multicolumn{4}{l}{ Amyloidosis $($ No (frequency)) } \\
\cline { 2 - 4 } & Absent & Present & $\chi^{2}$ \\
\hline 1.1 & $162(0.331)$ & $23(0.187)$ & $12.3(\mathrm{p}<0.001)$ \\
1.3 & $184(0.376)$ & $59(0.447)$ & $2.3(\mathrm{NS})$ \\
1.5 & $144(0.293)$ & $50(0.379)$ & $9.4(\mathrm{p}<0.01)$ \\
\hline
\end{tabular}

\section{Results}

CLINICAL FEATURES OF SUBJECTS BY SAA1 GENOTYPES

Data were assessed according to SAA1 genotype (table 1). There were no apparent differences in age, Steinbrocker functional class, duration of disease, use of corticosteroid (\%), DMARDS (\%), or immunosuppressive agents (\%). The SAA1 allele distributions in patients with amyloidosis were compared with those in subjects without (table 2). The allele 1.1 was negatively associated with amyloidosis $(p<0.001)$ while 1.5 was positively associated $(\mathrm{p}<0.01)$. There was no statistical difference in the 1.3 frequency between the groups.

INFLUENCE OF SAA1 GENOTYPES ON SAA AND CRP VALUES AND THE SAA/CRP RATIO

To assess the influence of the SAA1 allele on SAA and CRP values and on the SAA/CRP ratio, data from three homozygote groups were compared (table 3, fig 1. CRP concentrations did not differ between the three groups, whereas SAA concentrations and SAA/CRP ratios were significantly higher in the 1.5/1.5 than in the other groups.

FACTORS AFFECTING THE SAA/CRP RATIO Because the allelic bias on the SAA/CRP ratio was proved, subjects were divided into two groups according to the presence or absence of the SAA 1.5 allele and their data were analysed. Under these conditions the allele 1.2 was regarded as equivalent to the 1.5 as this allele is rare and has exon 3 structures, which distinguish the three common alleles, identical to

Table 3 Mean serum amyloid $A(S A A)$ and $C$ reactive protein (CRP) concentrations classified by SAA1 genotype

\begin{tabular}{lllll}
\hline $\begin{array}{l}\text { SAA1 } \\
\text { genotype }\end{array}$ & No & $S A A(\mathrm{mg} / \mathrm{l})$ & CRP $(\mathrm{mg} / \mathrm{l})$ & $S A A / C R P$ \\
\hline $1.1 / 1.1$ & 27 & 96 & 23 & 4.0 \\
$1.1 / 1.2$ & 3 & 185 & 35 & 8.7 \\
$1.1 / 1.3$ & 73 & 104 & 30 & 4.1 \\
$1.1 / 1.5$ & 55 & 193 & 29 & 6.8 \\
$1.2 / 1.3$ & 4 & 60 & 14 & 3.7 \\
$1.2 / 1.5$ & 3 & 195 & 11 & 14.8 \\
$1.3 / 1.3$ & 44 & 71 & 19 & 7.2 \\
$1.3 / 1.5$ & 78 & 105 & 16 & 11.0 \\
$1.5 / 1.5$ & 29 & $232^{\star}$ & 25 & $9.0 \dagger$ \\
Allele & & & & \\
$1.5(+)$ & 172 & $157 \ddagger$ & 22 & $9.1 \Phi$ \\
$1.5(-)$ & 144 & 93 & 26 & 5.0 \\
\hline
\end{tabular}

${ }^{\star} \mathrm{p}=0.022 v$ group $1.1 / 1.1, \mathrm{p}=0.009 v$ group $1.3 / 1.3$. $\mathrm{tp}=0.001 v$ group $1.1 / 1.1, \mathrm{p}=0.007 v$ group $1.3 / 1.3$. $\ddagger \mathrm{p}=0.005$, ๆ $\mathrm{p}<0.0001$ v group $1.5(-)$. 


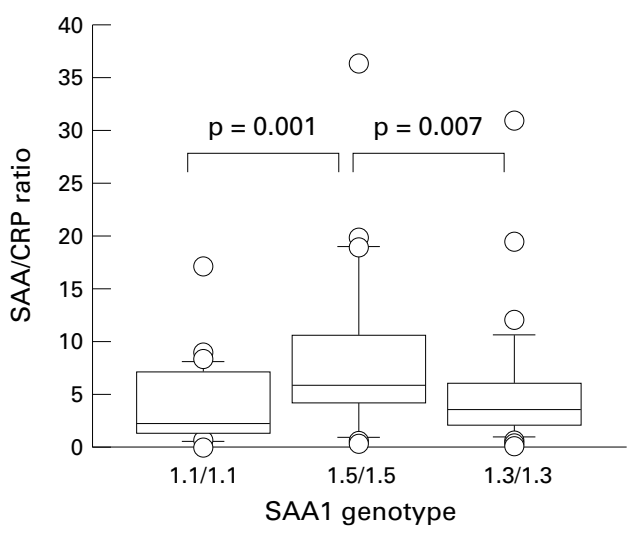

Figure 1 Box plots of serum amyloid $A / C$ reactive protein ( $S A A / C R P)$ ratios of the three $S A A 1$ homozygote groups. Boxes indicate 25th to 75 th centiles; lines in boxes, median values; and upper and lower bars, range from 0 to 100th centile. Subjects with 1.5/1.5 had a higher SAA/CRP ratio than the other two groups. No significant differences were noted between subjects with $1.1 / 1.1$ and 1.3/1.3 genotypes.

those of the 1.5 allele. The presence of the 1.5 allele was shown to have a positive influence on both SAA values and the SAA/CRP ratio (table 3).

Table 4 Influence of amyloidosis involvement and treatments on SAA/CRP* ratio. Results are shown as means

\begin{tabular}{|c|c|c|c|c|c|c|}
\hline & \multicolumn{2}{|c|}{ Amyloidosis } & \multicolumn{4}{|c|}{ Treatment (No) } \\
\hline & (No) & p Value & $C S^{\star}$ & p Value & $D M^{*}$ & $I S^{\star}$ \\
\hline \multicolumn{7}{|l|}{ All } \\
\hline Yes & $9.9(66)$ & 0.021 & $8.3(249)$ & $<0.0001$ & $6.7(252)$ & $6.0(107)$ \\
\hline No & $6.6(250)$ & & $3.7(67)$ & & $9.6(64)$ & $7.9(204)$ \\
\hline \multicolumn{7}{|c|}{$S A A 1.5$ (+) } \\
\hline Yes & $10.8(44)$ & 0.055 & $10.3(138)$ & $<0.0001$ & $8.0(137)$ & $7.9(62)$ \\
\hline No & $8.6(128)$ & & $4.6(34)$ & & $13.5(35)$ & $9.8(110)$ \\
\hline \multicolumn{7}{|c|}{$S A A 1.5(-)$} \\
\hline Yes & $8.2(22)$ & NS & $5.7(111)$ & 0.0003 & $5.0(115)$ & $3.4(45)$ \\
\hline No & $4.4(122)$ & & $2.8(33)$ & & $4.9(29)$ & $5.7(99)$ \\
\hline
\end{tabular}

${ }^{\star} \mathrm{SAA} / \mathrm{CRP}=$ serum amyloid $\mathrm{A} / \mathrm{C}$ reactive protein; $\mathrm{CS}=$ corticosteroid; $\mathrm{DM}=$ DMARDs; $\mathrm{IS}=$ immunosuppressive agents.

No significant differences were shown by the use of DM or IS

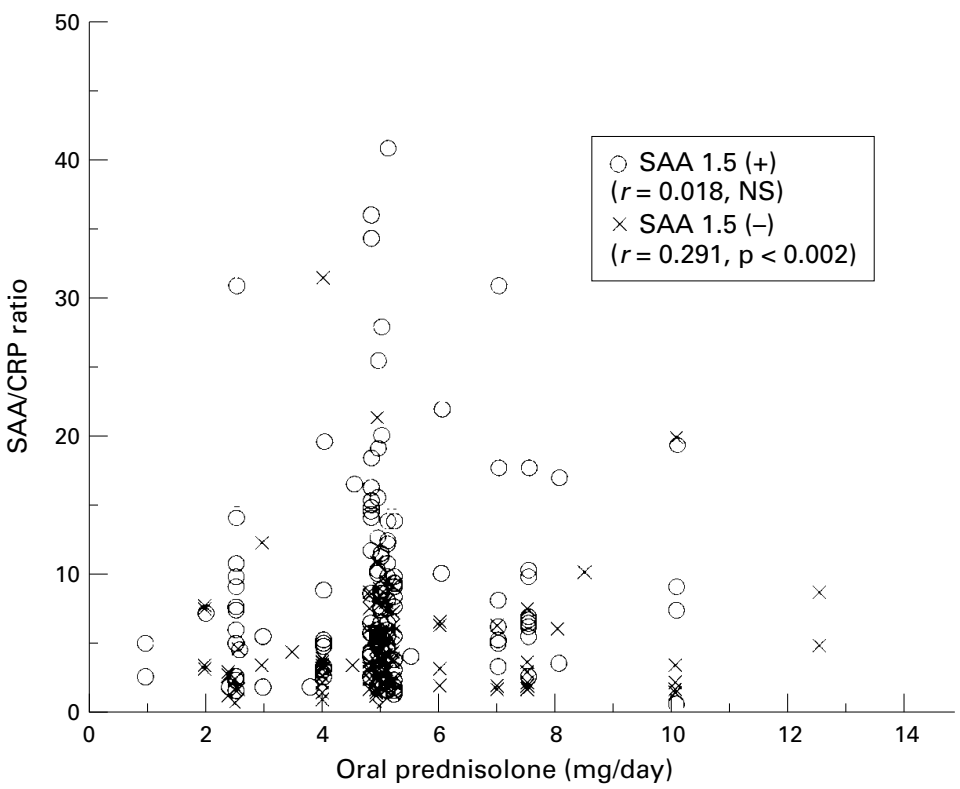

Figure 2 Relation between the serum amyloid $A / C$ reactive protein ( $S A A / C R P)$ ratio and dose of prednisolone. Significant correlation was observed in subjects with the allele SAA1.5 $(n=111)$, but not in those without it $(n=138)$. Data from five subjects were not included in the plot because of isolated data.
Age, sex, Steinbrocker functional class, or duration of the disease had no influence on the SAA/CRP ratio when either all the subjects or the groups divided by SAA1 allele were analysed (data not shown). The ratios were significantly higher $(\mathrm{p}<0.05)$ in patients with amyloidosis than in those without it when all the subjects were analysed (table 4). However, such differences did not reach significance in any of the divided groups. The use of DMARDs or immunosuppressive agents did not affect the SAA/CRP ratio, though the use of corticosteroids had a positive influence. This was seen both in all the subjects and in each of the divided groups (table 4). The SAA/CRP ratio correlated weakly with the dose of corticosteroid in the SAA1.5 group, but not in the allele negative group (fig 2).

\section{Discussion}

Two previous reports, including one by us, ${ }^{20} 21$ suggest that the SAA1.3 and SAA1.1 alleles are positive and negative risk factors for amyloidosis in Japanese patients with RA. One study ${ }^{21}$ showed an increased frequency, though not significant, of the SAA1.5 allele in subjects with amyloidosis. In the present study, similarly, a negative association of the allele 1.1 with amyloidosis was found. The increased frequency of 1.3, however, was not statistically significant. Instead, a significant association of 1.5 was shown. These controversial findings may be due to the subjects selected. Because this study did not aim at assessing susceptibility to amyloidosis, there may be some imbalance in the clinical profiles of the patients with or without amyloidosis. Nevertheless, the data show again the low frequency of 1.1. Perhaps, this may be a stronger genetic indication for amyloidogenesis in Japanese patients with RA than the high frequency of the allele 1.3.

In a previous report we described an allelic bias in plasma SAA concentrations of a healthy Japanese population sample. ${ }^{23}$ Our preliminary experiments with recombinant SAA1 isotypes suggested that the difference in plasma clearance among the allele-corresponding SAA phenotypes was responsible for this (unpublished findings). Whatever the mechanism, the possibility that such allelic bias is shown in subjects with abnormally raised SAA concentrations should be assessed. Because these concentrations change according to the inflammatory activity present when the blood sample is drawn, it was difficult to evaluate the relation between SAA values and SAA1 genotypes. Therefore, we introduced the SAA/CRP ratio. CRP correlated markedly with SAA in most of the inflammatory disorders. To date, no study has reported the participation of a genetic factor in producing a variation in serum CRP concentrations. By using this ratio, it was shown that SAA responds more sensitively than CRP to a kidney allograft rejection. ${ }^{5}$

The clinical profiles of the patients with RA in this study showed no apparent differences among the six major genotype groups. In addition, no statistical difference in CRP values was noted among the groups, suggesting that disease activity was also equally distributed. To 
examine the allelic influence on relative SAA values, the SAA/CRP ratios were compared in three homozygote groups: SAA1.1/1.1, SAA1.3/1.3, and SAA1.5/1.5. The results showed that SAA1.5 homozygotes had higher SAA/CRP ratios than the others. Consequently, the positive influence by allele 1.5 was confirmed not only in healthy (or minimally pathological) states but also in inflammatory conditions.

The above results indicate the need to evaluate SAA concentrations in the light of genetic information about subjects. A recent finding suggests that SAA is a better prognostic marker in early RA than CRP. ${ }^{26}$ Combining SAA1 genotypes and SAA values would be interesting. In this study we assessed what factors influenced the relative SAA values in subjects divided by SAA1 genotype. Because the number of each genotype, including three homozygote groups, was not enough for further analyses, we divided the subjects into two groups based on the presence of the allele 1.5.

As a result, the use of corticosteroid had an enhancing effect on the SAA/CRP ratio. The mechanism by which the SAA/CRP ratio is raised by corticosteroids is unknown. The agent may affect the synthesis of these proteins transcriptionally ${ }^{3}$ or post-transcriptionally, ${ }^{27}$ or it may have an effect by altering production of several inflammatory cytokines. Nevertheless, our findings support the concept that SAA responds more than CRP during the administration of corticosteroids. Dramatically high responses of SAA have been described for renal allograft rejection. ${ }^{68}$ Although this investigation did not assess the influence of cyclosporin, the agent most often used in renal transplantation, the high dose of corticosteroids, together with the effect of the SAA1 allele, may be responsible for this response. If the alteration of SAA levels is implicated in the development of amyloidosis, the propriety of giving corticosteroid treatment to patients at risk should concern investigators.

The subjects with amyloidosis in this study had higher SAA concentrations than those without it, but significance was borderline (table 4). The comparatively high prevalence of the SAA1.5 allele in this group may, in part, account for such an increase. The SAA/CRP ratios of subjects with amyloidosis did not differ from the ratio in those subjects without amyloidosis either in the groups divided by the presence of the SAA1.5 allele (table 4 ) or in the two homozygote groups, SAA1.3/1.3 and SAA1.5/1.5 (data not shown). Thus a strong linkage between the relative SAA values and presence of amyloidosis seems unlikely. However, as seen in Japanese subjects with the SAA1.1 allele, one cannot rule out the possibility that allele regulated SAA levels represent the allelic bias affecting their susceptibility to amyloidosis. To assess this further, other populations, such as white subjects, in whom SAA1 allele distribution and genetic background for amyloidogenesis differ from those of Japanese subjects, need to be analysed.

In conclusion, our study confirms a positive influence of the SAA1.5 allele and corticoster- oid treatment on relative SAA concentrations. These findings are important when evaluating SAA concentrations in diseases and when considering treatment in amyloidosis. Whether the allelic bias in amyloidogenicity is due to the allele regulated SAA levels or to other factors should be further investigated.

1 Kushner I. The acute phase response: an overview. Methods Enzymol 1988;163:373-83.

2 Malle E, deBeer FC. Human serum amyloid A (SAA) protein: a prominent acute-phase reactant for clinical practice. Eur J Clin Invest 1996;26:427-35.

3 Baumann H, Gauldie J. The acute phase response. Immunol Today 1994;15:74-80.

4 Steel DM, Whitehead AS. The major acute phase reactants: C-reactive protein, serum amyloid P component and serum amyloid A protein. Immunol Today 1994;15:81-8.

5 Maury CPJ. Comparative study of serum amyloid A protein in disease. Clin Sci 1985;68:233-8.

6 Yamada T. Serum amyloid A (SAA): a concise review of biology, assay methods and clinical usefulness. Clinical Chemistry and Laboratory Medicine 1999;37:381-8

7 Miwata H, Yamada T, Okada M, Kudo T, Kimura H, Morishima T. Serum amyloid A protein in acute viral infections. Arch Dis Child 1993;68:210-14.

8 Maury CPJ, Teppo AM. Comparative study of serum amyloid-related protein SAA, C-reactive protein, and $\beta_{2}$-microglobulin as markers of renal allograft rejection. Clin Nephrol 1984;22:284-92.

9 Smith JW, Colombo JL, McDonald TL. Comparison of serum amyloid $\mathrm{A}$ and C-reactive protein as indicators of lung inflammation in corticosteroid treated and noncorticosteroid treated cystic fibrosis patients. J Clin Lab Anal 1992;6:219-24

10 Dwulet FE, Benson MD. Amino acid structures of multiple forms of amyloid related serum protein SAA from a single individual. Biochemistry 1988;27:1677-82.

11 Betts JC, Edbrooke MR, Thakker RV, Woo P. The human acute-phase serum amyloid A gene family: structure, evolution and expression in hepatoma cells. Scand J Immunol 1991;34:471-82.

12 Sipe JD and Committee. Revised nomenclature for serum amyloid A (SAA). Amyloid 1999;6:67-70.

13 Yamada T. Analysis of serum amyloid A1 exon 4 polymorphism in Japanese population. Amyloid 2000;7:118-20

4 Husby G, Marhaug G, Dowton B, Sletten K, Sipe JD. Serum amyloid A (SAA): biochemistry, genetics and the pathogenesis of AA amyloidosis. Amyloid 1994;1:119-37.

15 Benson MD. Amyloidosis. In: Scriver CR, Beaudet AL, Sly WS, Valle DV, eds. The metabolic basis of inherited disease. 7 th ed. New York: McGraw-Hill, 1995:4159-91.

16 Laakso M, Mutru O, Isomaki HA, Koota K. Mortality from amyloidosis and renal diseases in patients with rheumatoid arthritis. Ann Rheum Dis 1986;45:663-7.

17 Kobayashi H, Tada S, Fuchigami T, Okuda Y, Takasugi K, Matsumoto T, et al. Amyloidosis in rheumatoid arthritis: diagnostic and prognostic value of gastroduodenal biopsy. Br J Rheumatol 1996;35:44-9.

18 Okuda Y, Takasugi K, Oyama T, Oyama H, Nanba S, Miyamoto T. Intractable diarrhoea associated with secondary amyloidosis in rheumatoid arthritis. Ann Rheum Dis 1997; 56:535-41.

19 Booth DR, Booth SE, Gillmore JD, Hawkins PN, Pepys MB. SAA1 alleles as risk factors in reactive systemic amyloidosis. Amyloid 1998;5:262-5.

20 Baba S, Masago SA, Takahashi T, Kasama T, Sugimura H, Tsugane $S$, et al. A novel allelic variant of serum amyloid A. SAA1 $\gamma$ : genomic evidence, evolution, frequency, and implication as a risk factor for reactive systemic amyloidoimplication as a risk factor for reactive

21 Okuda Y, Yamada T, Takasugi K, Takeda M, Nanba S, Onishi M, et al. Serum amyloid A (SAA) 1, SAA2 and apolipoprotein $\mathrm{E}$ isotype frequencies in rheumatoid arthritis patients with AA amyloidosis. Ryumachi 1999;39:3-10 (in apanese with English abstract)

22 Liepnieks JJ, Kluve-Beckerman B, Benson MD. Characterization of amyloid A protein in human secondary amyloidosis. The predominant deposition of serum amyloid A1. Biochim Biophys Acta 1995;1270:81-6.

23 Yamada T, Wada A, Itoh Y, Itoh K. Serum amyloid A1 alleles and plasma SAA concentration. Amyloid 1999;6:199-204.

24 Arnett FC, Edworthy SM, Bloch DA, McShane DJ, Fries Arnett FC, Edworthy SM, Bloch DA, McShane DJ, Fries
JF, Cooper NS, et al. The American Rheumatism Association 1987 revised criteria for the classification of rheumatoid arthritis. Arthritis Rheum 1988;31:315-24.

25 Yamada T, Nomata Y, Sugita O, Okada M. A rapid method for measuring serum amyloid A protein by latex agglutination nephelometric immunoassay. Ann Clin Biochem 1993;30:72-6.

26 Cunnane G, Grehan S, Geoghegan S, McCormack C, Shields D, Whitehead AS, et al. Serum amyloid A in the assessment of early inflammatory arthritis. J Rheumatol 2000;27:58-63.

27 Lozanski G, Jiang SL, Samols D, Kushner I. C-reactive protein and serum amyloid A mRNA stability following induction by cytokines. Cytokine 1996;8:534-40. 